\title{
A Hybrid Particle Swarm Optimization Based on Symmetric Distribution and Simulated Annealing
}

\author{
Xueyan Li \\ Faculty of Information Engineering, City College of Wuhan University of Science and Technology, \\ Wuhan 430083, China \\ 25233666@qq.com
}

Keywords: particle swarm optimization; multimodal function; radial symmetric; simulated annealing

\begin{abstract}
The performance of particle swarm optimization (PSO) is limited by its local minima, defects and poor precision. To solve this problem, we present a hybrid adaptive particle swarm optimization. Two approaches, symmetric distribution and simulated annealing algorithm, have been used to improve the PSO algorithm. Firstly, the position of particle is updated by means of a radial symmetric function in the center. Secondly, the simulated annealing algorithm is employed to describe the mechanism. Finally, the improved PSO algorithm in our research is verified to be feasible and effective by comparing with the current well-known methods.
\end{abstract}

\section{Introduction}

Particle swarm optimization is a swarm intelligence technique developed by Kennedy and Eberhart in 1995 [1], inspired by the paradigm of birds flocking. In recent years, particle swarm optimization has been used increasingly as an effective technique for solving complex and difficult optimization problems, such as multi-objective optimization, training neural network and emergent system identification [2-4]. However, it suffers from premature convergence especially in high dimensional problem, and it is hard to escape from the local optimization. This shortcoming has restricted the wide application of PSO. As a result, accelerating convergence rate and avoiding local optima have become the most important and appealing goals in PSO research [5].

Therefore, it is extremely significant to solve these problems mentioned above. In recent years, a variety of improved particle swarm optimization have appeared. Some of these variations choose to change the initialization method of PSO. For example, the initialization method has been changed by M. pant [6,7], Plow PSO[8]. Other types of improved PSO include adjusting parameters, such as hybrid PSO[9-12]. In order to avoid convergence and local optima, we address a new approach to improving particle swarm optimization. Our approach has been validated by four common complex multimodal and unmodal benchmark functions. The simulation results demonstrate that the proposed method about PSO is efficient to resolve complex numerical function optimization problems.

This paper is organized as follows: section 2 describes the standard PSO algorithm; section 3 proposes the hybrid PSO algorithm and describes it in detail;in section 4, experiments on four benchmark functions are provided; conclusions are given in section 5.

\section{The Theory of Particle Swarm Optimization}

The particle swarm optimization is one of the most Swarm intelligences, and it is inspired by the paradigm of birds or fish, and this algorithm has been introduced by James Kennedy and Russell Eberhart in 1995, PSO is a randomly optimal algorithm, and it can solve optimization problems. The Detailed steps are as follows: the population initialized by random particles, and calculating their fitness values, finding the personal best value, and global-best value, and the iteration. Every particle update their velocity using formula 1 ,and their position using formula 2 . Each time a particle finds a better position , its location is stored in memory. In other words, the algorithm works on the social behavior of particles in the swarm. As a result, it finds the global-best position by simply adjusting 
their own position toward its own best location and toward the best particle of the entire swarm at each step.

$$
\begin{aligned}
& v_{i j}(t+1)=w v_{i j}(t)+c_{1} r_{1}\left(\text { pbest }_{i j}(t)-x_{i j}(t)\right) \\
& +c_{2} r_{2}\left(\text { gbest }_{g j}(t)-x_{i j}(t)\right) \\
& x_{i j}(t+1)=x_{i j}(t)+v_{i j}(t+1)
\end{aligned}
$$

Where $t$ indicates the iteration number; $w$ is the inertia weight; $r_{1}$ and $r_{2}$ are two random vectors range 0 to $1 ; i=1,2,3, \ldots N, N$ is the warm size ; $j=1,2,3, \ldots . D, D$ means the dimension of Searching place; $\mathrm{v}$ indicates the velocity of each particle; $\mathrm{x}$ means the position of each particle. We call this contains the inertia weight particle swarm optimization for IWPSO.

The velocity vector of each particle is affected by their personal experiences and global particle factors 。 The position of each particle in the next iteration is calculated by adding its velocity vector to its current position.

In 2002, a constriction named $\lambda$ coefficient is used to prevent each particle from exploring too far away in the range of min a max, since $\lambda$ applies a suppression effect to the oscillation size of a particle over time. This method, constricted PSO as suggested by Clerc and Kennedy, is used with $\lambda$ set it to 0.7298, according to the formula [11].

$$
\lambda=\frac{2}{\left|2-\varphi-\sqrt{\varphi^{2}-4 \varphi}\right|}, \text { where } \varphi=\varphi_{1}+\varphi_{2}
$$

\section{Improved Adaptive Particle Swarm Optimization}

\section{Analysis of Improved Adaptive Particle Swarm Optimization}

PSO algorithm is population-based, which means a lot of particles search the best position and have little specific knowledge. This advantage makes it more robust than other EA. However, PSO lacks the ability of global search in the end. In most cases, PSO easily falls into local optimum when it is used to solve some too complicated and complex problems. So it is very significant to find a solution to the global optimum of PSO algorithm.

On one hand, setting parameter in PSO is also an important issue. The choice of algorithm parameters has a greater impact on the efficiency of the algorithm. Different patterns of change may lead to different parameters to optimize results. Parameters should make changes to increase dynamic performance of the algorithm which has a certain smoothness. So the algorithm behavior under different parameters have a good transition. In practice, it is difficult to define these rules. Therefore, how to choose the right parameters to achieve optimal efficiency is the key to solve a problem in the PSO algorithm. On the other hand, in the searching process, the appropriate selection operator can be effectively beyond the local minimum area.

\section{The Principle of Improved Algorithm}

On the basis of the standard PSO, we have conducted the following two improvements: first, we use inverse multi-quadratic function of the randomization approach to guide the particle escape from local optimum strategy. The inverse multi-quadratic function makes the change that the PSO algorithm has a wider distribution of search and is easy to generate a random number from the origin. The inverse multi-quadratic function is the center of radial symmetry, the formula is defined as (4), and its waveform is shown in Fig.1. Updated particle position calculated as formula (5), $\eta$ is symmetrically distributed random variables obey.

$$
\begin{aligned}
& \varphi(x)=\left(x^{2}+c^{2}\right)^{1 / 2} \quad \mathrm{c}>0 ; \mathrm{x} \in \mathrm{R} \\
& x_{i j}(t+1)=x_{i j}(t)(1+0.6 \eta)
\end{aligned}
$$




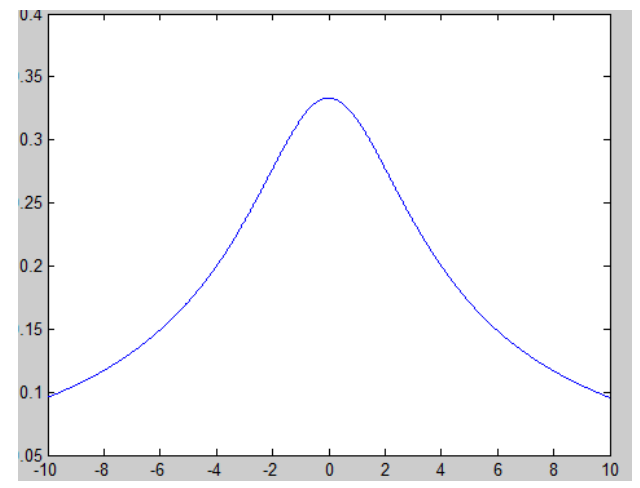

Fig.1. An inverse multi-quadratic function.

Second, as the simulated annealing ideas can accept worse solution, so it can help out local maximum particle and find the global optimum. Simulated annealing algorithm is a probability-based random search optimization algorithm; simulated annealing algorithm has strong global search capability. Combining the particle swarm optimization algorithm with simulated annealing algorithm can play a complementary role, and improve effectively the performance of the algorithm.

Based on the above two considerations, this paper proposes a new method based on multi-quadratic function of the adaptive particle swarm optimization.

\section{Description of Algorithm}

According to the introduction of algorithm, procedure has described in the previous section.

Step 1: Initializing the swarm: $\mathrm{T}_{0}$

a. Set the initial temperature $\left(T_{0}\right)$, end temperature $\left(T_{e}\right)$ and current temperature $\left(T_{c}\right)$, first step set

b. Initializing the size of particle, calculating the global optimum(gbest) and the personal optimum(pbest), first step set gbest=pbest.

c. Set the learning factor $\mathrm{c}_{1}$ and $\mathrm{c}_{2}$.

Step2: Calculating the fitness value of each particle.

Step3: Immunization for each particle: add random perturbations to each individual to choose a new group using multi-quadratic function.

Step4: Compare the particle of current fitness value with its own individual fitness value, if the current fitness value is better than individual's, then set the best personal value to the current fitness value, and set the location of individual extreme position for the current.

Step5: Compare each particle's fitness value and global extreme, if the particle's fitness value is more than the global fitness value, then modify the global extreme value for the current particle's fitness value. On the contrary, according to the Metropolis criterion, accepting worse solution as a certain probability.

Step6: Annealing operation: $T_{k}=\beta * T_{0}$.

Step7: update the particle position and velocity, the formula as above (1)(5).

Step8: Conditions if the algorithm terminates, then the algorithm stops, otherwise the transfer step2.

\section{Simulation Results}

\section{Definition of Functions}

In order to verify the validity of the improved PSO algorithm, we focus on a set of well-known benchmark functions with many local minima used in other Evolutionary Algorithms. Four functions taken from[8] are given in Table 2. These benchmark functions in our Table 2 are numbered from $\mathrm{f}_{1}$ to $\mathrm{f}_{4}$.

\section{Experimental Settings}

Detailed settings is shown in Table 1. 


\section{Experimental Results}

The results are shown in Table 3 as the average optimal fitness using the four PSOs over 30 independent runs respectively, and the best results are marked in boldface. On the multimodal functions, the experimental results of the three PSOs are analyzed as follows: the efficiency of PSO is the lowest, the second is IWPSO, and our proposed PSO offers the best. The reason lies that the mechanism of Simulated annealing speeds up the implementation of the PSO algorithm.

\section{Conclusions}

In this paper, a comparison between four optimization techniques, four test functions have been performed to illustrate our improvement . Simulation results show that our proposed algorithm effectively solves the PSO algorithm in the presence of local optimum, stagnation and shock, and effectively improve the efficiency of the algorithm.

Table 1. The experimental settings.

\begin{tabular}{|l|l|l|l|l|l|l|l|}
\hline Algorithm & size & $\begin{array}{c}\text { Simulated } \\
\text { annealing } \\
\text { factor }\end{array}$ & $\begin{array}{c}\text { Initial } \\
\text { temperature }\end{array}$ & $\mathrm{c}_{1}$ & $\mathrm{c}_{2}$ & $\mathrm{w}_{\max } / \mathrm{w}_{\mathrm{mi}}$ & iter $_{\max }$ \\
\hline PSO & 40 & --- & --- & 2 & 2 & $0.9 / 0.4$ & 5000 \\
\hline IWPSO & 40 & --- & --- & 2.05 & 2.05 & $0.95 / 0.4$ & 5000 \\
\hline $\begin{array}{l}\text { The } \\
\text { improved } \\
\text { PSO }\end{array}$ & 40 & 0.95 & 10000 & 2 & 2 & $0.9 / 0.4$ & 5000 \\
\hline
\end{tabular}

Table 2. Definition of functions.

\begin{tabular}{|l|l|l|l|}
\hline $\begin{array}{c}\text { Name of } \\
\text { function }\end{array}$ & \multicolumn{1}{|c|}{ Function Definition } & \multicolumn{1}{|c|}{ Range } & \multicolumn{1}{|c|}{$\begin{array}{c}\text { Minimum } \\
\text { value }\end{array}$} \\
\hline Spherical & $f_{1}(x)=\sum_{i=1}^{n} x_{i}^{2}$ & {$[-5.12,5.12]$} & 0 \\
\hline Schewefel & $f_{2}(x)=\sum_{i=1}^{n}-x_{i} \sin \left(\sqrt{\left|x_{i}\right|}\right)$ & {$[-500,500]$} & -8329.658 \\
\hline Griewank & $f_{3}(x)=\frac{1}{4000} \sum_{i=1}^{n} x_{i}^{2}-\prod_{i=1}^{n} \cos \left(\frac{x_{i}}{\sqrt{i}}\right)+1$ & {$[-600,600]$} & 0 \\
\hline Rastrigin & $f_{4}(x)=\sum\left[x_{i}^{2}-10 \cos \left(2 \pi x_{i}+10\right)\right]$ & {$[-5.12,5.12]$} & 0 \\
\hline
\end{tabular}

Table 3. The Result of The Experiment Comparing With Other PSOs.

\begin{tabular}{|l|l|l|l|l|}
\hline \multicolumn{1}{|c|}{ Function } & \multicolumn{1}{c|}{$\mathbf{f}_{\mathbf{1}}$} & \multicolumn{1}{c|}{$\mathbf{f}_{\mathbf{2}}$} & \multicolumn{1}{c|}{$\mathbf{f}_{\mathbf{3}}$} & $\mathbf{f}_{\mathbf{4}}$ \\
\hline PSO & $3.7701 \mathrm{e}-10$ & -6853.55301 & 0.0105771 & 5.718810 \\
\hline IWPSO & $5.99233 \mathrm{e}-23$ & -7003.3077 & 0.009334 & 4.998105 \\
\hline $\begin{array}{l}\text { The } \\
\text { proposed } \\
\text { PSO }\end{array}$ & $5.0030511 \mathrm{e}-68$ & $\mathbf{- 8 1 0 0 . 5 8 8 9 1}$ & $\mathbf{0 . 0 0 8 6 9 8}$ & $\mathbf{3 . 9 0 5 5 6}$ \\
\hline
\end{tabular}

\section{Acknowledgement}

In this paper, the research was sponsored by the Education Department Foundation of Hubei Province of China under Grant No. B2015362 . 


\section{References}

[1] Bergh F V d. A new locally convergent particle swarm optimizer[C]. IEEE International Conference on systems,Man and Cybernetics,2002.

[2] Shi Y,Eberhart R C. A Modified Particle Swarm Optimizer[C]. Proceedings of the IEEE International Conference on Evolutionary Computation, Piscataway, NJ: IEEE Press, 1998,69-73.

[3] Yuhui Shi, Russell C. Eberhart .Empirical Study of Particle Swarm Optimization[C]. Proceedings of the IEEE Congress on Evolutionary Computation CEC'99 .1999,3:1945-1950.

[4] Ingber L. Very fast simulated annealing [J]. Mathematical Computer Modeling ,1989,12:967 973.

[5] Dalvinder Mangal. Hammerstein Model Identification Based On Instrumental Variable and Least Square Methods[J]. Journal of Emerging Trends and Technology in Computer Science,2013,2(1):44-48.

[6] Jun Sun, Bin Feng, Wenbo Xu. Particle Swam Optimization with Particles Having Quantum Behavior[C]. Proceedings of the IEEE Congress on Evolutionary Computation CEC2004,2004,2:19-23.

[7] Kenndy J,Eberhart R C.Particle Swarm Optimization[C].Proceedings of IEEE International Conference on Neural Networks,1995,1942-1948.

[8] TsallisC,StarioloDA.Physical,1996,A233:395

[9] Hbabaee, An improve PSO based Hybrid Algorithms. Management and Service Science(MASS), International Conference , 2011

[10] Ghanad N K, Ahmadi S. Combination of PSO Algorithm and Naive Bayesian Classification for Parkinson Disease Diagnosis[J]. Advances in Computer Science: an International Journal, 2015, 4: 119-125.

[11] Jordehi A R. Enhanced leader PSO (ELPSO): A new PSO variant for solving global optimisation problems[J]. Applied Soft Computing, 2015, 26: 401-417.

[12] Shriya S, Sharma R S, Sumit S, et al. Directed Search-based PSO Algorithm and Its Application to Scheduling Independent Task in Multiprocessor Environment[C]/Proceedings of the 4th International Conference on Frontiers in Intelligent Computing: Theory and Applications (FICTA) 2015. Springer India, 2016: 23-31. 